\title{
Assessment of Collateral Status by Dynamic CT Angiography in Acute MCA Stroke: Timing of Acquisition and Relationship with Final Infarct Volume
}

(DI.R. van den Wijngaard, (D) Holswilder, (D).J.H. Wermer, (D). Boiten, (D)A. Algra, (DD.W.J. Dippel, (D).W. Dankbaar, (D)B.K. Velthuis, (D)A.M.M. Boers, (D)C.B.L.M. Majoie, and (D).A.A. van Walderveen

\begin{abstract}
BACKGROUND AND PURPOSE: Dynamic CTA is a promising technique for visualization of collateral filling in patients with acute ischemic stroke. Our aim was to describe collateral filling with dynamic CTA and assess the relationship with infarct volume at follow-up.
\end{abstract}

MATERIALS AND METHODS: We selected patients with acute ischemic stroke due to proximal MCA occlusion. Patients underwent NCCT, single-phase CTA, and whole-brain CT perfusion/dynamic CTA within 9 hours after stroke onset. For each patient, a detailed assessment of the extent and velocity of arterial filling was obtained. Poor radiologic outcome was defined as an infarct volume of $\geq 70 \mathrm{~mL}$. The association between collateral score and follow-up infarct volume was analyzed with Poisson regression.

RESULTS: Sixty-one patients with a mean age of 67 years were included. For all patients combined, the interval that contained the peak of arterial filling in both hemispheres was between 11 and 21 seconds after ICA contrast entry. Poor collateral status as assessed with dynamic CTA was more strongly associated with infarct volume of $\geq 70 \mathrm{~mL}$ (risk ratio, 1.9; 95\% Cl, 1.3-2.9) than with single-phase CTA (risk ratio, 1.4; 95\% Cl, 0.8-2.5). Four subgroups (good-versus-poor and fast-versus-slow collaterals) were analyzed separately; the results showed that compared with good and fast collaterals, a similar risk ratio was found for patients with good-but-slow collaterals (risk ratio, $1.3 ; 95 \% \mathrm{Cl}, 0.7-2.4)$.

CONCLUSIONS: Dynamic CTA provides a more detailed assessment of collaterals than single-phase CTA and has a stronger relationship with infarct volume at follow-up. The extent of collateral flow is more important in determining tissue fate than the velocity of collateral filling. The timing of dynamic CTA acquisition in relation to intravenous contrast administration is critical for the optimal assessment of the extent of collaterals.

ABBREVIATION: CS = collateral score

Cllateral vessel status is an important predictor of final infarct size and clinical outcome in patients with a stroke due to acute proximal anterior circulation occlusions. ${ }^{1,2}$ Leptomeningeal collaterals can provide a detour for blood to reach the ischemic territory of an occluded artery, thereby sustaining tissue at

Received September 17, 2015; accepted after revision January 4, 2016. From the Departments of Radiology (I.R.v.d.W., G.H., M.A.A.v.W.), Neurology (M.J.H.W.), and Clinical Epidemiology (A.A.), Leiden University Medical Center, Leiden, the Netherlands; Department of Neurology (I.R.v.d.W., J.B.), Medical Center Haaglanden, the Hague, the Netherlands; Department of Neurology and Neurosurgery (A.A.), Brain Center Rudolf Magnus, and Department of Radiology (J.W.D., B.K.V.), University Medical Center Utrecht, Utrecht, the Netherlands; Department of Neurology (D.W.J.D.), Erasmus University Medical Center, Rotterdam, the Netherlands; and Departments of Radiology (A.M.M.B., C.B.L.M.M.) and Biomedical Engineering and Physics (A.M.M.B.), Academic Medical Center, Amsterdam, the Netherlands.

DUST was supported by the Dutch Heart Foundation (2008T034) and the NutsOhra Foundation (0903-012). The MR CLEAN trial was supported by the Dutch Heart Foundation and by unrestricted grants from AngioCare, Covidien/ev3, Medac/Lamepro, and Penumbra.

I.R. van den Wijngaard and G. Holswilder contributed equally to this work. risk for a longer time. ${ }^{1,3,4}$ DSA is considered the criterion standard for visualization of the collateral circulation. However, DSA is currently reserved for part of the thrombectomy procedures and has no role in the diagnostic work-up of patients with acute ischemic stroke.

In clinical practice, imaging of collaterals is often performed with single-phase CTA. Single-phase CTA visualizes the cerebral circulation at only a single moment in time. The information captured in this snapshot depends on the timing of the CTA acquisition after contrast injection, which may lead to inaccurate estimation of the collateral circulation. ${ }^{1,5,6}$ Dynamic CTA is increasingly investigated for the visualization of collaterals in acute

Please address correspondence to Ido van den Wijngaard, MD, Department of Radiology, Leiden University Medical Center, PO Box 9600, 2300 RC Leiden, the Netherlands; e-mail: wijngaardido@gmail.com

- Indicates open access to non-subscribers at www.ajnr.org

$\equiv$ Indicates article with supplemental on-line appendix and tables.

Indicates article with supplemental on-line photos.

http://dx.doi.org/10.3174/ajnr.A4746

AJNR Am J Neuroradiol 37:1231-36 Jul 2016 www.ajnr.org 
ischemic stroke. ${ }^{7-9}$ Dynamic CTA can be derived from CT perfusion datasets and provides time-resolved images of the arterial, parenchymal, and venous phases. Not only the extent but also the velocity of collateral filling can be evaluated with dynamic CTA.

The aim of this study was to assess dynamic CTA as a method for imaging the collateral circulation in patients with acute ischemic stroke. We devised a grading system that gives information on the extent and velocity of pial arterial filling in a time-resolved manner. We compared this new method with collateral assessment on single-phase CTA in relation to infarct volume at follow-up.

\section{MATERIALS AND METHODS Study Population}

Patients from the Leiden University Medical Center were selected from the Dutch Acute Stroke Study (DUST) and the Multicenter Randomized CLinical trial of Endovascular treatment for Acute ischemic stroke in the Netherlands (MR CLEAN). Protocol details of these clinical studies with inclusion and exclusion criteria have been published before. ${ }^{10,11}$ Patients with an acute ischemic stroke with a proximal MCA occlusion (M1 or M2 segments) were included, with or without occlusion of the ICA. All patients underwent multimodal CT imaging including NCCT, singlephase CTA, and whole-brain CT perfusion/dynamic CTA at presentation. Adult patients were included up to 9 hours after symptom onset in DUST. For the MR CLEAN trial, initiation of endovascular treatment had to be possible within 6 hours after symptom onset. Clinical data were retrieved from the study data bases. Informed consent was obtained from patients who participated in these clinical studies.

\section{CT Image Acquisition}

For CT image acquisition, a 320-section multidetector CT scanner, Aquilion ONE (Toshiba Medical Systems, Tokyo, Japan) was used, resulting in a whole-brain CTP coverage from 320-detector arrays of $0.5 \mathrm{~mm}$. All patients underwent a standard scanning protocol at presentation, including NCCT, single-phase CTA from the aortic arch to the vertex, and whole-brain CT perfusion/ dynamic CTA. For the whole-brain CT perfusion/dynamic CTA, 19 volumes were obtained during 1 minute. (See the On-line Appendix for more details about image acquisition.)

\section{Image Analysis}

Radiologic data were independently assessed by a trained neuroradiologist and a trained neurologist, who were given information regarding the clinical symptoms only. At admission, early ischemic changes on NCCT were evaluated with the ASPECTS. ${ }^{12}$ To evaluate the extent of arterial occlusion, we applied the clot burden score. $^{13}$

NCCT was performed at 1- to 5-day follow-up for assessment of infarct volume (or earlier in case of severe clinical deterioration, ie, $\geq 4$-point increase in the NIHSS compared with baseline). Infarct volumes at follow-up were independently assessed and provided to us by the DUST and MR CLEAN investigators. ${ }^{14}$ Poor radiologic outcome was defined as an infarct volume of $\geq 70 \mathrm{~mL}$.

\section{Collateral Score}

On single-phase CTA, the collaterals were graded with the collateral score (CS) proposed by Tan et al. ${ }^{15}$ This "Tan CS" uses a 4-point grading system, ranging from 0 to 3 , with which it grades vessel filling in the territory of the occluded artery to assess collateral circulation. A Tan CS of zero indicates absent filling, in which no vessels are visible within the occluded MCA territory. A Tan CS of 1 indicates arterial contrast filling of $\leq 50 \%$ of the occluded MCA territory. A score of 2 was given when contrast filling was present in $>50 \%$ but $<100 \%$ of the occluded MCA territory, and a score of 3, when contrast filling was present in $100 \%$ of the occluded MCA territory.

On dynamic CTA, a more extensive grading system was devised to assess the collateral circulation; this grading system used the same 4-point grading system of the CS of Tan et al, ${ }^{15}$ but now applied in 4 separate brain regions. Each of the 19 volumes of the dynamic CTA acquisitions was evaluated to obtain information about the velocity of contrast filling and the extent of the contrastfilled arteries with time. Vessel filling was graded in 4 separate brain regions, areas $1 \mathrm{a}, 1 \mathrm{~b}, 2 \mathrm{a}$, and $2 \mathrm{~b}$, with the objective of obtaining a detailed description of vessel filling with time as visualized by dynamic CTA. Area 1 indicated the ipsilateral (ie, affected) hemisphere, and area 2 indicated the contralateral (ie, unaffected) hemisphere. Both areas were divided into 2 separate areas, in which $a$ indicated the part of area 1 or 2 below the body of the caudate nucleus and $b$ indicated the part above the body of the caudate nucleus. All 4 areas were provided with a Tan CS from 0 to 3. To obtain information about the extent of vessel filling with time for the ipsilateral hemisphere, we added the Tan CS for area 1 a to the Tan CS of area $1 \mathrm{~b}$, resulting in a total score ranging from 0 to 6 . The same was performed for the contralateral hemisphere, by adding a Tan CS of area $2 \mathrm{a}$ to area $2 \mathrm{~b}$. A total extent of filling from 0 to 3 corresponded to arterial filling of $\leq 50 \%$ of the hemisphere and a total extent of filling from 4 to 6 denoted filling of $>50 \%$ (see On-line Fig 1 for examples).

The velocity of filling was evaluated by calculating the duration in seconds of contrast arrival at the ICA until maximal contrast enhancement for each hemisphere separately. Subsequently, the difference in the duration of filling between the ipsilateral and contralateral hemisphere was calculated. The median difference in optimal filling time for all patients was used as cutoff value to discern fast-from-slow ipsilateral filling. The combined data of the extent and velocity of filling enabled subgroup analysis (Online Fig 2). For each subgroup, we defined the optimal assessment interval as the interval that contained the 3 highest average collateral scores in both the affected and unaffected hemispheres.

\section{Statistical Analysis}

Statistical analysis was performed with SPSS statistics (Version 22; IBM, Armonk, New York). The associations between clinical and radiologic characteristics and a poor radiologic outcome were analyzed with univariable Poisson regression and were expressed as risk ratios. Accompanying 95\% confidence intervals were used to describe their precision. Interobserver agreement was assessed by using the Cohen $\kappa$ statistic. The additional prognostic value of collaterals as assessed by using dynamic CTA over the assessment by using single-phase CTA on radiologic outcome was analyzed 
by using logistic regression models. In the first model, the dichotomized collateral score assessed on single-phase CTA was used to predict radiologic outcome. In a second model, the dichotomized collateral score assessed on dynamic CTA was added to the first model. Subsequently, the potential improvement of the second model with respect to the first model was compared by using the likelihood ratio test. Graphs were computed by calculating the mean filling of all patients grouped according to extent of collateral filling and for different time points in seconds after contrast arrival at the ICA. Standard error of the mean was indicated by error bars representing \pm 1 standard error.

\section{RESULTS}

\section{Patients}

From July 2010 until July 2014, 70 patients were eligible, of whom 61 had follow-up imaging available for measurement of the final infarct volume. The mean age of the study participants was 67 , the median NIHSS score was 15, and 27 patients (44\%) were women. The median time from symptom onset to multimodel CT imaging was 64 minutes. Most patients $(61 \%, n=37)$ received intravenous thrombolysis, only $5 \%(n=3)$ underwent mechanical thrombectomy without intravenous thrombolysis and 23\% $(n=$ 14) received a combined treatment of intravenous thrombolysis and mechanical thrombectomy. The remaining $11 \%(n=7)$ did not receive any treatment (On-line Table 1). At follow-up, 33 patients $(54 \%)$ had a poor radiologic outcome (infarct volume, $\geq 70 \mathrm{~mL}$ ).

\section{Collateral Assessment}

Interobserver agreement for assessment of collateral extent with dynamic CTA was excellent ( $\kappa=0.88, n=61$ ). Collateral filling assessed with single-phase CTA showed grade 0 (absent) in $8 \%$ $(n=5)$, grade 1 (moderate) in $61 \%(n=37)$, grade 2 (good) in $30 \%(n=18)$, and grade 3 (excellent) in $2 \%(n=1)$. Collateral filling scores were distributed differently when evaluated by dynamic CTA. Most patients had, for each region (ie, below and above the body of the caudate nucleus), either a collateral score of 2 indicating $>50 \%$ vessel filling (level $a: n=30,49 \%$; level $b: n=$ $23,38 \%$ ) or a collateral score of 3 indicating complete vessel filling (level $a: n=21,34 \%$; level $b: n=23,38 \%$ ). The remaining patients were graded with a collateral score of 1 indicating $<50 \%$ vessel filling (level $a: n=10,16 \%$; level $b: n=15,25 \%$ ) (On-line Fig 2).

For the entire study population, dynamic CTA analysis showed that the median time difference between optimal filling of the healthy hemisphere compared with the affected hemisphere was 4.5 seconds. Fast filling was defined as optimal filling within 4.5 seconds after optimal filling in the unaffected hemisphere. Combining the extent and velocity of filling resulted in 4 different subgroups: good-and-fast collaterals, good-but-slow collaterals, poor-but-fast collaterals, and poor-and-slow collaterals (On-line Fig 3).

Patterns of vessel filling with dynamic CTA were described for the contralateral (ie, unaffected) and the ipsilateral (ie, affected) hemisphere as shown in Fig $1 A$. The unaffected hemisphere reached complete arterial filling (ie, score of 6) on average at 14 seconds after contrast entry at the ICA, while the ipsilateral hemi- sphere showed average optimal collateral filling (ie, filling score of 4) 16 seconds after contrast entry at the ICA. For all patients combined, the interval that contained the 3 highest average collateral filling scores for both hemispheres was between 11 and 21 seconds after ICA contrast entry; the optimal assessment interval was 10 seconds.

Subgroups with fast filling showed a narrow optimal assessment interval of 8 seconds (ie, between 11 and 19 seconds after ICA contrast entry), while subgroups with slow attainment of optimal ipsilateral filling had a broader optimal assessment interval of 14 seconds (range, 11-25 seconds in case of poor extent) and 10 seconds (range, 11-21 seconds in case of good extent) (Fig $1 B-E$ ).

\section{Dynamic versus Single-Phase CTA}

In comparison with collateral status as evaluated on single-phase CTA, collateral status assessed with dynamic CTA had a stronger association with poor radiologic outcome, as shown in the Table. Poor collaterals (ie, filling in $\leq 50 \%$ of the occluded MCA territory) as assessed with dynamic CTA showed an increased risk of poor radiologic outcome compared with good collaterals (ie, $>50 \%$ filling), with a risk ratio of 1.9 (95\% CI, 1.3-2.9).

The dichotomized collateral score assessed on single-phase CTA showed a less strong relationship; poor collaterals showed a risk ratio of 1.4 (95\% CI, $0.8-2.5)$ on poor radiologic outcome. (Table; risk ratios of other clinical and radiologic variables are presented in On-line Table 2). With logistic regression, the prediction of radiologic outcome by using model 1 , containing dichotomized collateral scores as assessed on single-phase CTA, was compared with the prediction of model 2 with the addition of dichotomized collateral scores as assessed on dynamic CTA. The likelihood ratio test showed a significant increase in predictive value between model 1 and model $2(P<.01)$. Characterizing patients based on the extent and velocity of filling assessed with dynamic CTA indicated an increased risk of poor radiologic outcome with decreasing collateral status. Poor collaterals and slow filling showed an increased risk compared with good collaterals and fast filling, with a risk ratio of 2.0 (95\% CI, 1.0-3.8). Poor collaterals and fast filling had an almost identical effect, with a risk ratio of 1.9 (95\% CI, 1.1-3.2). Good collaterals and slow filling showed a risk for poor radiologic outcome identical to that of good collaterals and the fast-filling group, with a risk ratio of 1.3 (95\% CI, 0.7-2.4) (Table). Patients who received different treatments or had different rates of reperfusion were equally represented in the groups with different collateral scores (On-line Tables 3 and 4).

\section{DISCUSSION}

This study showed that dynamic CTA enables detailed description of the collateral circulation by assessing contrast flow through the cerebral arteries during multiple time points in patients with acute ischemic stroke. Collateral assessment with dynamic CTA had a stronger relationship with infarct volume at follow-up than assessment with single-phase CTA. Although multiple studies have indicated that collateral status as evaluated on single-phase CTA can predict both clinical and radiologic outcomes, ${ }^{1,4,16}$ relatively few studies have been performed with dynamic CTA. ${ }^{5,17}$ 

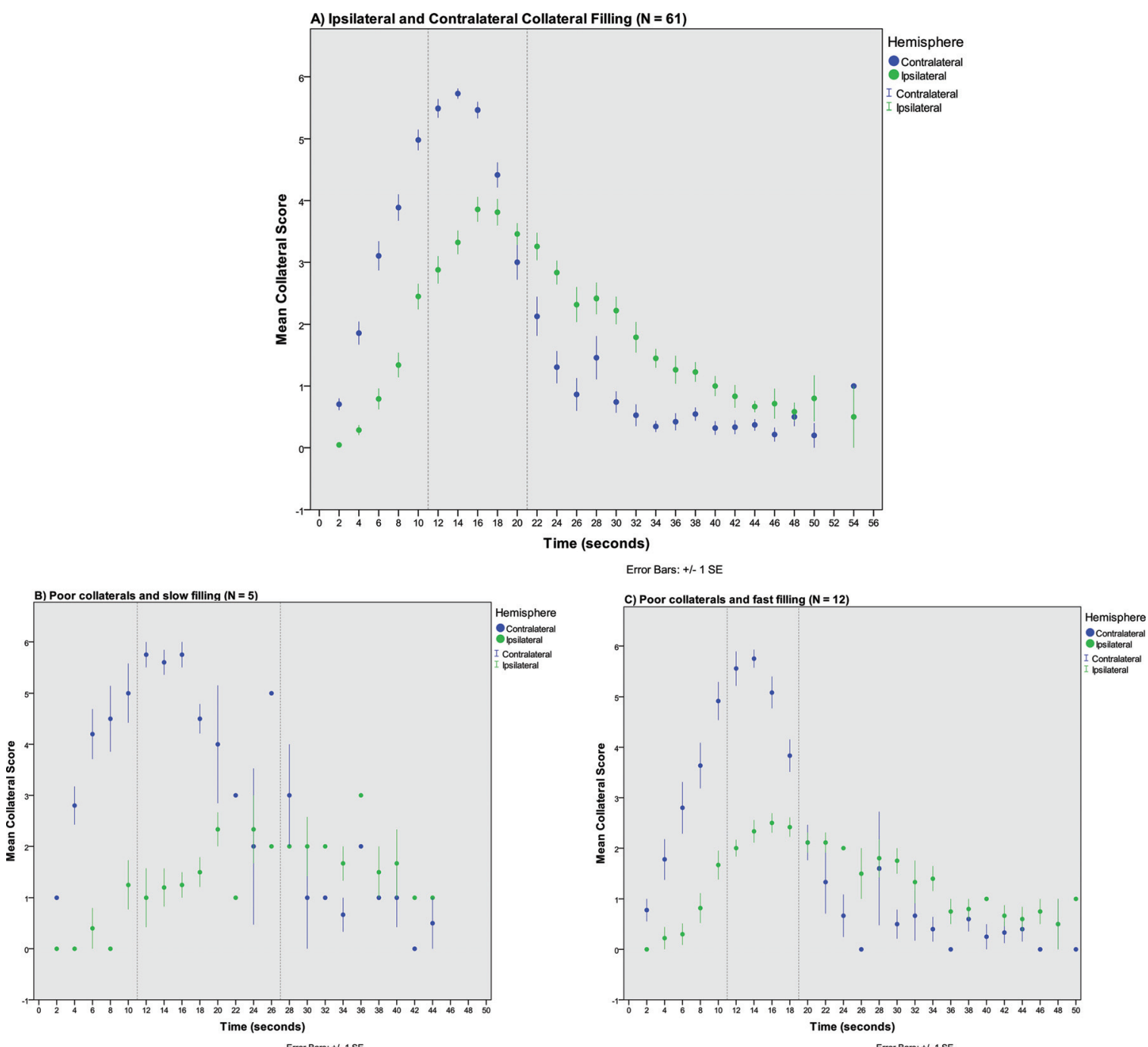

Emror Bars: +1-1 SE

Emor Bars: + +1 1 SE
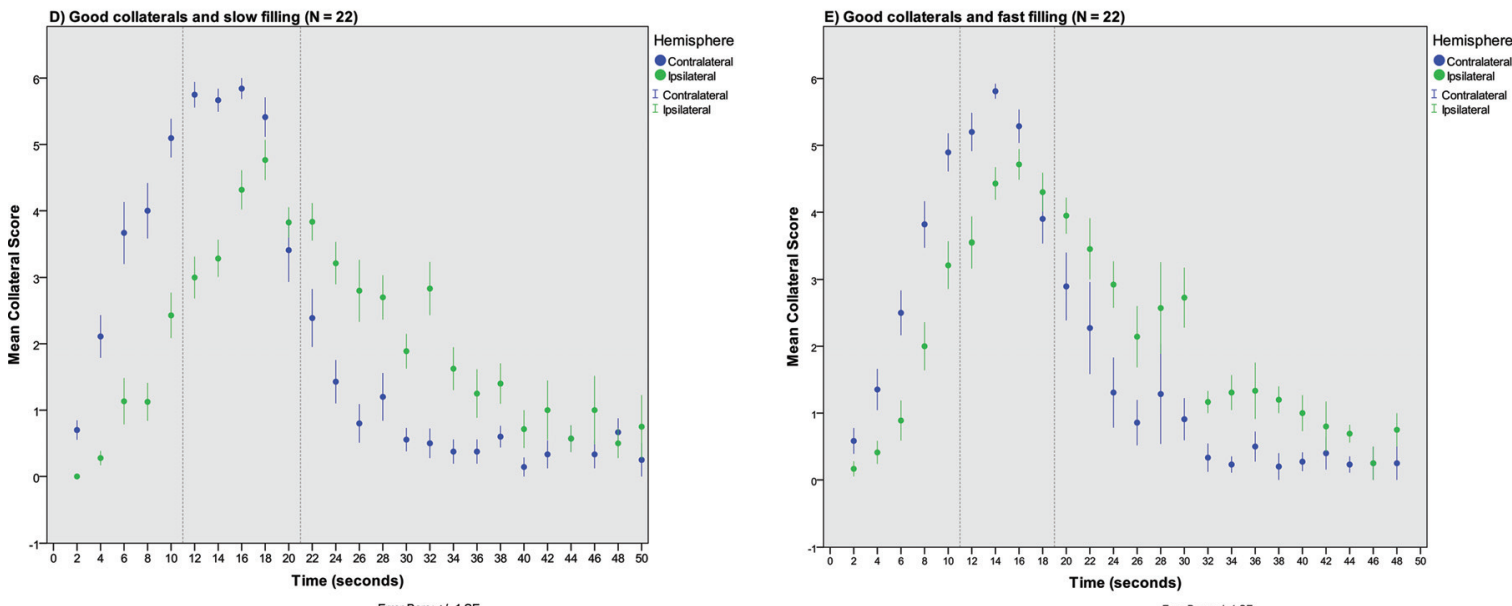

FIG 1. Patterns of contralateral and ipsilateral vessel filling for all patients and for 4 different subgroups. $A$, Ipsilateral and contralateral vessel filling of all patients $(n=61)$. Time in seconds after contrast arrival in the ICA was calculated for each volume and averaged for multiple patients for each different time point in seconds. Green dots represent mean filling with time after contrast arrival for the ipsilateral (ie, affected) hemisphere. Blue dots represent mean filling with time after contrast arrival in the ICA for the unaffected contralateral hemisphere. Error bars represent 1 standard error of the mean (1 standard error). Dotted vertical lines indicate the optimal assessment interval, which is defined by the presence of the 3 highest average collateral scores in both the affected and unaffected hemispheres. B, Patients with poor collateral filling of $<50 \%$ of the ischemic territory and slow filling, defined as ipsilateral filling reaching optimal filling $>4.5$ seconds later than contralateral optimal vessel filling $(n=5)$. C, Patients with poor collateral filling of $<50 \%$ of the ischemic territory but with fast filling within 4.5 seconds after contralateral optimal vessel filling $(n=12)$. D, Patients with good collateral filling of $>50 \%$ and slow filling $(n=22)$. $E$, Patients with good collateral filling of $>50 \%$ and fast filling $(n=22)$. 


\begin{tabular}{|c|c|c|c|}
\hline & $\begin{array}{c}\text { Poor Outcome/ } \\
\text { Characteristic } \\
\text { Present }(n / N)(\%)\end{array}$ & $\begin{array}{l}\text { Poor Outcome/ } \\
\text { Characteristic } \\
\text { Absent }(n / N)(\%)\end{array}$ & $\begin{array}{c}\text { Risk Ratio } \\
(95 \% \mathrm{Cl})\end{array}$ \\
\hline \multicolumn{4}{|l|}{ Single-phase CTA } \\
\hline Poor extent of collateral filling ${ }^{a}$ & $25 / 42(60 \%)$ & $8 / 19(42 \%)$ & $1.4(0.8-2.5)$ \\
\hline \multicolumn{4}{|l|}{ Dynamic CTA, extent of filling } \\
\hline CS 0-1 & - & - & - \\
\hline CS 2 & $5 / 5(100 \%)$ & $7 / 19(37 \%)^{\mathrm{b}}$ & $2.7(1.5-4.9)$ \\
\hline CS 3 & $9 / 12(75 \%)$ & $7 / 19(37 \%)^{b}$ & $2.0(1.0-4.0)$ \\
\hline CS 4 & $8 / 13(62 \%)$ & $7 / 19(37 \%)^{b}$ & $1.7(0.8-3.5)$ \\
\hline CS 5 & $4 / 12(33 \%)$ & $7 / 19(37 \%)^{b}$ & $0.9(0.4-2.4)$ \\
\hline \multicolumn{4}{|l|}{ Dynamic CTA } \\
\hline Poor extent of collateral filling ${ }^{a}$ & $14 / 17(82 \%)$ & $19 / 44(43 \%)$ & $1.9(1.3-2.9)$ \\
\hline \multicolumn{4}{|c|}{ Dynamic CTA, extent and timing of filling } \\
\hline Poor collaterals and slow filling & $4 / 5(80 \%)$ & $9 / 22(41 \%)^{c}$ & $2.0(1.0-3.8)$ \\
\hline Poor collaterals and fast filling & $23 / 30(77 \%)$ & $9 / 22(41 \%)^{c}$ & $1.9(1.1-3.2)$ \\
\hline Good collaterals and slow filling & $14 / 26(54 \%)$ & $9 / 22(41 \%)$ & $1.3(0.7-2.4)$ \\
\hline
\end{tabular}

cause the division of the MCA is variable and because we wanted to take the extent and velocity of all vessel filling into account, we chose a recognizable brain structure on axial images, namely the body of the caudate nucleus, which was not dependent on vessel filling. Although we acknowledge that this may be only an approximation of the true division of vascular territories, it resulted in a consistent distinction of upper and lower MCA territories over all dynamic CTA volumes. Moreover, we have demonstrated high interobserver agreement.

Another limitation of our study was the relatively low number of patients (ie, $<25 \%$ ) who underwent DSA, which prevented a direct comparison of dynamic CTA with DSA. Another limitation is the applicability of this grading

Dynamic CTA resulted in higher collateral scores than collateral scores assessed with single-phase CTA, which indicates underestimation when depicting the collateral circulation at 1 time point only. This outcome is in accordance with that of a previous study in which patients with acute ischemic stroke were found to show higher filling scores when imaged with multiphase CTA than with single-phase CTA. ${ }^{17,18}$ Multiphase CTA is an alternative method for collateral assessment by using only a few strategic time points. Both multiphase and dynamic CTA have shown superior results of collateral assessment for predicting clinical outcome compared with conventional single-phase CTA. ${ }^{18,19} \mathrm{Be}-$ cause collateral assessment with multiphase CTA has already been used for patient selection in a large thrombectomy trial, ${ }^{20}$ assessment with dynamic CTA also looks promising for implementation in future endovascular treatment trials and clinical practice. A potential advantage of dynamic CTA over multiphase CTA is that dynamic CTA is constructed from CT perfusion data, which have additional value for predicting clinical outcome. ${ }^{21}$

Furthermore, dynamic CTA provided us with contrast-filling data to compute an average contrast flow through the cerebral arteries of the affected and the unaffected hemispheres of patients with acute ischemic stroke. We found that collaterals become increasingly apparent from the early-to-late phases up to a point at which the affected hemisphere shows stronger opacification than the unaffected hemisphere due to more rapid contrast medium washout of the unaffected hemisphere. On average, the affected hemisphere reaches a lower extent of optimal filling at a later time point in comparison with the unaffected hemisphere. Recently, the arteriovenous phase of dynamic CTA was found to be optimal for collateral assessment to predict follow-up infarct volume. ${ }^{7}$ Although we did not assess the venous circulation for this study, our results also indicate that the timing of CTA acquisition for optimal collateral assessment of the affected hemisphere is always after the peak arterial phase of the unaffected hemisphere.

A limitation of our study is that our dynamic collateral scoring system is new and requires validation by future research. The grading system was a modification of the one used by Souza et al, ${ }^{22}$ whose scores were based on the division of M2 branching. Be- system in clinical practice, because assessment of dynamic CTA in all 19 volumes is time-consuming. However, for fast interpretation in clinical practice, maximum intensity projections of all 19 volumes can be created and displayed chronologically. This feature creates angiography-like movies of time-resolved MIPs. As discussed before, optimization of the conventional CTA scanning with $\geq 3$ strategic time points (ie, multiphase CTA) is another alternative and could be sufficient for the assessment of the complete collateral circulation. ${ }^{16}$ Future prospective studies with larger sample sizes are needed to compare dynamic CTA with multiphase CTA.

Our results show that the velocity of collateral filling does not seem to determine radiologic outcome as much as the extent of filling; these findings are in line with a previous study. ${ }^{5}$ We demonstrated that optimal image acquisition is performed within the 11- to 21-second range after ICA contrast entry. We observed that the period containing the peak filling phases of both hemispheres (ie, the optimal assessment interval) differed depending on the collateral status based on extent and velocity of filling. For patients with a poor and slow collateral circulation, it seems less important to image at a later time point because vessel filling will remain suboptimal during the entire contrast bolus passage. However, in case of slow-but-good collateral filling, it is important to have an extra image acquisition moment at a later time point. Patients with slow-but-good collateral circulation might be incorrectly labeled as having a poor collateral circulation at the point of peak arterial filling in the unaffected hemisphere. Thus, additional image acquisitions at later time points are required.

\section{CONCLUSIONS}

Dynamic CTA is a useful method for the evaluation of the collateral circulation in patients with acute ischemic stroke. It has a strong association with radiologic outcome at follow-up and provides detailed information on the extent and velocity of contrast bolus filling through the cerebral vasculature. This study indicates that it might be important to extend the CTA acquisition for a longer time. Because collateral assessment on dynamic CTA is 
time-consuming, this acquisition can possibly be reduced to only 3 or 4 strategic image-acquisition phases to simplify and speed up assessment of collaterals in patients with acute MCA stroke.

Disclosures: Marieke J.H. Wermer-RELATED: Grant: supported by a personal grant from the Netherlands Organisation for Scientific Research (NWO/ZonMw VENI grant) and the Netherlands Heart Foundation (2011T055).* Diederik W.J. DippelUNRELATED: Consultancy: Erasmus MC received funds from Stryker for consultations*; The MR CLEAN trial was partly funded by the Dutch Heart Foundation* and by unrestricted grants from AngioCare BV, ${ }^{*}$ Medtronic/Covidien/ev3,* Medac/ Lamepro,* Penumbra, ${ }^{\star}$ Stryker,* Top Medical/Concentric.* Jan W. DankbaarRELATED: Grant: supported by a personal grant from the Netherlands Heart Foundation (2012T061). Birgitta K. Velthuis—RELATED: Grant: Dutch Heart Foundation (grant No. 2008T034)*; UNRELATED: Payment for Lectures (including service on Speakers Bureaus): Philips Healthcare. * Charles B.L.M. Majoie—RELATED: Grant: Dutch Heart Foundation*; UNRELATED: Payment for Lectures (including service on Speakers Bureaus): Stryker.* *Money paid to the institution.

\section{REFERENCES}

1. Menon BK, Smith EE, Modi J, et al. Regional leptomeningeal score on CT angiography predicts clinical and imaging outcomes in patients with acute anterior circulation occlusions. AJNR Am J Neuroradiol 2011;32:1640-45 CrossRef Medline

2. Singer OC, Berkefeld J, Nolte $\mathrm{CH}$, et al. Collateral vessels in proximal middle cerebral artery occlusion: the ENDOSTROKE study. Radiology 2015;274:851-58 CrossRef Medline

3. Liebeskind DS, Tomsick TA, Foster LD, et al; IMS III Investigators. Collaterals at angiography and outcomes in the Interventional Management of Stroke (IMS) III trial. Stroke 2014;45:759-64 CrossRef Medline

4. Lima FO, Furie KL, Silva GS, et al. Prognosis of untreated strokes due to anterior circulation proximal intracranial arterial occlusions detected by use of computed tomography angiography. JAMA Neurol 2014;71:151-57 CrossRef Medline

5. Frölich AM, Wolff SL, Psychogios MN, et al. Time-resolved assessment of collateral flow using 4D CT angiography in large-vessel occlusion stroke. Eur Radiol 2014;24:390-96 CrossRef Medline

6. Higashida RT, Furlan AJ, Roberts H, et al; Technology Assessment Committee of the American Society of Interventional and Therapeutic Neuroradiology, Technology Assessment Committee of the Society of Interventional Radiology. Trial design and reporting standards for intra-arterial cerebral thrombolysis for acute ischemic stroke. Stroke 2003;34:e109-37 CrossRef Medline

7. Beyer SE, Thierfelder KM, von Baumgarten L, et al. Strategies of collateral blood flow assessment in ischemic stroke: prediction of the follow-up infarct volume in conventional and dynamic CTA. AJNR Am J Neuroradiol 2015;36:488-94 CrossRef Medline

8. Latchaw RE, Alberts MJ, Lev MH, et al; American Heart Association Council on Cardiovascular Radiology and Intervention, Stroke Council, and the Interdisciplinary Council on Peripheral Vascular Disease. Recommendations for imaging of acute ischemic stroke: a scientific statement from the American Heart Association. Stroke 2009;40:3646-78 CrossRef Medline

9. Menon BK, O’Brien B, Bivard A, et al. Assessment of leptomeningeal collaterals using dynamic CT angiography in patients with acute ischemic stroke. J Cereb Blood Flow Metab 2013;33:365-71 CrossRef Medline

10. van Seeters T, Biessels GJ, van der Schaaf IC, et al; DUST investigators. Prediction of outcome in patients with suspected acute ischaemic stroke with CT perfusion and CT angiography: the Dutch acute stroke trial (DUST) study protocol. BMC Neurol 2014;14:37 CrossRef Medline

11. Fransen PS, Beumer D, Berkhemer OA, et al; MR CLEAN Investigators. MR CLEAN, a multicenter randomized clinical trial of endovascular treatment for acute ischemic stroke in the Netherlands: study protocol for a randomized controlled trial. Trials 2014;15:343 CrossRef Medline

12. Barber PA, Demchuk AM, Zhang J, et al. Validity and reliability of a quantitative computed tomography score in predicting outcome of hyperacute stroke before thrombolytic therapy: ASPECTS Study Group-Alberta Stroke Programme Early CT Score. Lancet 2000; 13:1670-74 Medline

13. Puetz V, Dzialowski I, Hill MD, et al; Calgary CTA Study Group. Intracranial thrombus extent predicts clinical outcome, final infarct size and hemorrhagic transformation in ischemic stroke: the clot burden score. Int J Stroke 2008;3:230-36 CrossRef Medline

14. Boers AM, Marquering HA, Jochem JJ, et al; MR CLEAN investigators. Automated cerebral infarct volume measurement in follow-up noncontrast CT scans of patients with acute ischemic stroke. AJNR Am J Neuroradiol 2013;34:1522-27 CrossRef Medline

15. Tan JC, Dillon WP, Liu S, et al. Systematic comparison of perfusion-CT and CT-angiography in acute stroke patients. Ann Neurol 2007;61:533-43 CrossRef Medline

16. Maas MB, Lev MH, Ay H, et al. Collateral vessels on CT angiography predict outcome in acute ischemic stroke. Stroke 2009;40:3001-05 CrossRef Medline

17. Smit EJ, Vonken EJ, van Seeters $T$, et al. Timing-invariant imaging of collateral vessels in acute ischemic stroke. Stroke 2013;44:2194-99 CrossRef Medline

18. Menon BK, d'Esterre CD, Qazi EM, et al. Multiphase CT angiography: a new tool for the imaging triage of patients with acute ischemic stroke. Radiology 2015;275:510-20 CrossRef Medline

19. van den Wijngaard IR, Boiten J, Holswilder G, et al. Impact of collateral status evaluated by dynamic computed tomographic angiography on clinical outcome in patients with ischemic stroke. Stroke 2015;46:3398-404 CrossRef Medline

20. Goyal M, Demchuk AM, Menon BK, et al; ESCAPE Trial Investigators. Randomized Assessment of Rapid Endovascular Treatment of Ischemic Stroke. N Engl J Med 2015;372:1019-30 CrossRef Medline

21. Borst J, Berkhemer OA, Roos YB, et al; MR CLEAN Investigators and Affiliations. Value of computed tomographic perfusion-based patient selection for intra-arterial acute ischemic stroke treatment. Stroke 2015;46:3375-82 CrossRef Medline

22. Souza LC, Yoo AJ, Chaudhry ZA, et al. Malignant CTA collateral profile is highly specific for large admission DWI infarct core and poor outcome in acute stroke. AJNR Am J Neuroradiol 2012;33: 1331-36 CrossRef Medline 